\title{
Computation of pile kinematic bending moments in non-homogeneous soil profiles. Testing the validity of a simplified Vs30-equivalent homogeneous medium.*
}

\author{
Guillermo M. Álamo, Juan J. Aznárez, Luis A. Padrón, Orlando Maeso \\ Instituto Universitario de Sistemas Inteligentes y Aplicaciones Numéricas en Ingeniería (SIANI), \\ Universidad de Las Palmas de Gran Canaria, Edificio Central del Parque Científico y Tecnológico, \\ Campus Universitario de Tafira, 35017 Las Palmas de Gran Canaria, Spain \\ [guillermo.alamo / juanjose.aznarez / luis.padron / orlando.maeso] @ulpgc.es
}

\begin{abstract}
The influence of the soil profile on the maximum kinematic bending moments of pile foundations is investigated in this work. For this purpose, power-law, linear and equivalent homogeneous profiles based on real boreholes are selected together with characteristic dimensions of pile foundations. The equivalence between the homogeneous and variable profiles is established in terms of the average shear wave velocity $V_{s, 30}$ suggested by the codes for site classification. Envelopes of maximum kinematic bending moments are computed through the application of the standard frequency-domain method and accelerograms corresponding to real earthquake events. The pile harmonic response is computed through an efficient linear-elastic numerical model based on the reciprocity theorem in elastodynamics and the use of Green's functions for the layered half space. In this model, piles are considered as Timoshenko's beam elements and treated as load lines within the soil formulation. The obtained results show a large influence of the variability of the soil profile on the pile maximum kinematic bending moments both for single pile and pile groups. At the pile head, the homogeneous assumption underestimates the pile response, while the opposite effect is found along the pile shaft.
\end{abstract}

\section{Keywords:}

pile foundations; non-homogeneous soil; seismic response; kinematic bending moments; pilesoil-pile interaction; boundary integral formulation

\section{Introduction}

The seismic response of pile foundations has been an interesting topic of study in the soilstructure interaction field for the last decades. Based on field evidence and experimental tests $[1,2,3,4]$, it has been found that the seismically-induced pile internal forces (kinematic forces) could be as important for the pile failure as the ones induced by the vibration of the supported structure (inertial forces).

\footnotetext{
*Pre-print of the paper published in SOIL DYNAMICS AND EARTHQUAKE ENGINEERING, 131, 106062 (2020), DOI: $10.1016 /$ j.soildyn.2020.106062
} 
The effects of the soil profile characteristics on kinematic bending moments of pile foundations have received great attention during the last years. Especially, numerous research works $[5,6,7,8,9,10,11,12,13,14,15]$ have focused on the problem of piles in soils that present several layers with sharply differing stiffness. In this particular case, critical kinematic bending moments are found around the layers interface, being sometimes larger that the ones present at the pile head. In this regard, some authors $[9,10,11,12,13,14]$ have proposed design-oriented formulas to estimate those maximum bending moments at the layer interface, as well as the ones produced atop of piles with fixed-rotation head conditions. Because most of the previous studies were based on single pile foundations, Dezi and Poulos [15] proposed expressions of correction factors that can be used in order to incorporate the group effects in those estimated bending moments. On the other hand, the analysis of the pile kinematic bending moments in soils with continuous non-homogeneity has received less attention. One example is the work of Di Laora and Rovithis [16] in which single piles embedded in several variable-with-depth soil profiles were studied based on a Winkler approach, deriving expressions for the pile-head kinematic bending and the kinematic active length of the pile. The calculation of this active length was further developed by Karatzia and Mylonakis [17]. With the aid of these expressions, Mucciacciaro and Sica [18] interpreted the kinematic pile bending in a problem where the soil non-homogeneity was a consequence of the soil stiffness degradation during the seismic load.

Firstly proposed by Borcherdt and Glassmoyer [19] and Borcherdt [20], the average shear wave velocity of the upper $30 \mathrm{~m}$, i.e. $V_{s, 30}$, is the parameter used for soil site classification in many seismic codes $[21,22]$. Its definition is based on the time that the shear wave takes to travel along these $30 \mathrm{~m}$. However, some studies have questioned its use as a proxy for seismic amplification $[23,24,25]$, especially in cases of shallow velocity inversion [26], or for predictions involving large $(T>1 \mathrm{~s})$ periods [27]. Recently, alternative or complementary parameters to the $V_{s, 30}$ have been proposed for soil site classification, such as the average shear wave velocity over the upper $z \mathrm{~m}$ (being $z$ from $5-30 \mathrm{~m}$ ) $[28,29]$ or up to a rigid bedrock [30], the site fundamental frequency $[29,30,31]$, the thickness of the soil deposit [30], or the S-wave impedance [32]. Regarding the use of the $V_{s, 30}$ as a parameter for the characterization of the soil medium when studying the pile kinematic bending in non-homogeneous media, Stacul and Squeglia [33] discussed its relevance in their recent work. They found that, for stating whether kinematic bending must be considered in pile seismic design, a threshold value in terms of the average shear wave velocity along the active length of the pile was more effective than the one based on $V_{s, 30}$ suggested by seismic codes.

The present paper aims at further analysing the behaviour of the pile kinematic bending moments in non-homogeneous soils. Two objectives are established: 1) to compare the maximum kinematic bending moments of piles embedded in different continuously variable soil profiles, and 2 ) to test whether the assumption of a homogeneous medium with the average shear wave velocity $V_{s, 30}$ could be used to estimate the response obtained for the non-homogeneous profile. For these purposes, results corresponding to two continuously variable soil profiles with similar mean shear wave velocities, as well as one homogeneous profile, are compared in terms of envelopes of maximum kinematic bending moments.

\section{Problem statement}

\subsection{Foundation definition}

With the aim of studying the kinematic response of floating piles embedded in non-homogeneous soils, different pile foundations and terrains will be considered. The study is focused on the response of the single pile, as its behaviour is representative of the one of pile groups. However, 
the influence of group effects on kinematic bending moments is also addressed considering square pile groups formed by identical elements whose heads are linked together through an infinitelyrigid massless cap.

A wide range of pile geometries is considered for the present study. Pile lengths $L$ from 10 to $50 \mathrm{~m}$ (increments of $10 \mathrm{~m}$ ) and pile diameters $d$ from 0.1 to $0.5 \mathrm{~m}$ (increments of $0.1 \mathrm{~m}$ ) and from 0.5 to $2 \mathrm{~m}$ (increments of $0.25 \mathrm{~m}$ ) are combined in order to obtain useful results from an engineering point of view. For the group configurations, centre-to-centre separation distance ratios $s / d$ from 2 to 10 (increments of 2 ) are considered.

The material properties are selected assuming solid cross-section concrete piles: elasticity modulus $E=30 \mathrm{GPa}$, density $\rho=2500 \mathrm{~kg} / \mathrm{m}^{3}$, and Poisson's ratio $\nu=0.2$. These values are also representative of hollow steel piles modelled by equivalent soil cross-section properties. No material damping is considered for the piles.

Two head conditions are assumed in the analysis of the single pile: free and fixed-rotation. The free head condition models piles that are not linked to any superstructure (e.g., piles used as barriers) and can also be representative of the response of piles in a group with a hinged pile-cap union. On the other hand, the fixed-rotation at head condition is commonly used to model the restriction imposed by the superstructure to the foundation rotation. In this case, the results of fixed-rotation head single piles are closely related to the ones of the piles in a group with a fixed pile-cap union, even if no rotation restriction is imposed to the cap motion. Note that the fixedrotation condition is an idealized situation for studying only the kinematic response of the pile without considering the inertial effects of the supported structure. Eventually, the combination of the kinematic and inertial responses can lead to maximum head bending moments larger than the ones obtained for the independent extreme conditions (i.e., fixed-rotation head for the foundation or fixed-base for the superstructure).

\subsection{Soil profiles definition}

Piles are assumed to be founded in ground types D and E of the ASCE [22] classification (corresponding to types $\mathrm{C}$ and $\mathrm{D}$ of the Eurocode [21] classification). Two sets of soil profiles, one for each ground type, are considered for the analysis. Each set is constituted by three soil profiles: two variable-with-depth profiles and a depth-independent profile.

For the definition of the variable soil profiles, the expressions proposed by Wang and Wang [34] for the evolution with depth of the soil shear wave velocity are considered. These expressions were obtained by a fitting procedure of data from real boreholes of California and Japan soils. Linear and power-law expressions were proposed for each region and each ground type. In the present study, both the linear and power-law profiles for the California region are used. The expressions of the California soils are selected over the ones of Japan because the firsts are also representative of other seismically active regions in the world (e.g., the Mediterranean zone).

On the other hand, for each ground type, the homogeneous profile is defined by a constant soil shear wave velocity coincident with the mean shear wave velocity in the upper $30 \mathrm{~m}\left(V_{s, 30}\right)$ of the variable soil profiles. Note that, due to the elastodynamic nature of the fitting procedure used by Wang and Wang [34], the average shear wave velocities of the linear and power-law profiles virtually coincide, being $V_{s, 30}=252$ and $157 \mathrm{~m} / \mathrm{s}$ for the studied profiles of ground types $\mathrm{D}$ and $\mathrm{E}$, respectively. This average shear wave velocity is defined so that the shear wave takes the same time to travel through the first $30 \mathrm{~m}$ of the variable-with-depth and homogeneous media, and it is used in the seismic codes for site classification. Its expression for a continuously 
Type D

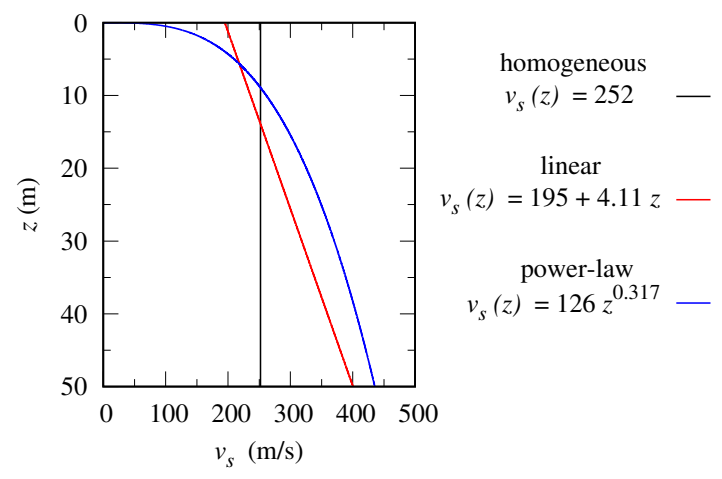

Type E

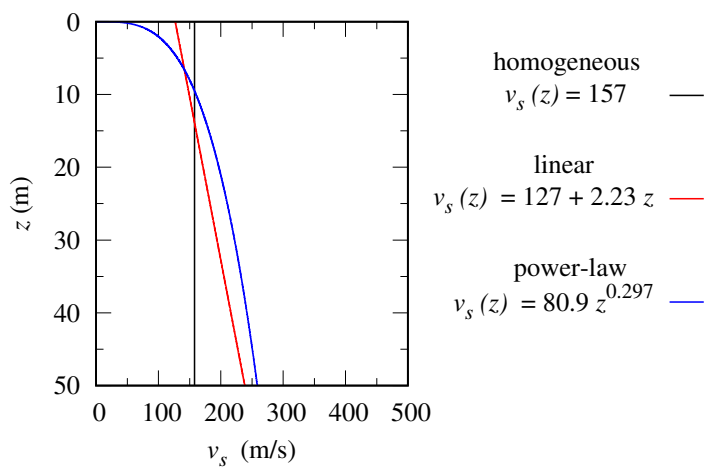

Figure 1: Evolution-with-depth of the soil profiles corresponding to the two ground types (according to ASCE Site Class Classification [22]). Expressions extracted from Wang and Wang [34] (units in $\mathrm{m} / \mathrm{s}$ ).

variable profile can be extrapolated from the one of a layered soil [21, 22] as:

$$
V_{s, 30}=\frac{30}{\int_{0}^{30} \frac{\mathrm{d} z}{v_{s}(z)}}
$$

Figure 1 illustrates the evolution with depth of the shear wave velocity corresponding to the three studied profiles per ground type. Note the singular nature of the power-law profile, which presents a zero value of the shear wave velocity at surface level and will therefore magnify the effects of strong variations of the soil stiffness near the ground surface. The rest of soil properties are assumed to be depth-independent and are defined by a soil density $\rho_{s}=1750$ $\mathrm{kg} / \mathrm{m}^{3}$, a Poisson's ratio $\nu_{s}=0.4$, and a soil hysteretic damping coefficient $\beta_{s}=2.5 \%$. In order to model the continuously-varying profiles with the proposed methodology (see Section 3), they are discretized into piecewise homogeneous layers with a thickness of $0.125 \mathrm{~m}$ along the first 50 $\mathrm{m}$ of the soil (as depicted by Fig. 1). Below this depth, the soil properties are kept constant and equal to the ones at $50 \mathrm{~m}$ depth for the underlying half space. These values of layer thickness and maximum depth are obtained from a convergence analysis.

\section{$2.3 \quad$ Excitation definition}

Piles are assumed to be excited by vertically propagating shear (S) waves. Sets of seven accelerograms per ground type are used to obtain the envelopes of maximum bending moments of the piles. The accelerograms are extracted from the PEER Ground Motion Database [35], corresponding to seismic events produced in the California region with magnitudes between 6 and 7. Only signals measured at stations located over soils of ground types D and E, respectively as the studied one, are used. For reproducibility's sake, Table 1 identifies the used accelerograms, indicating the Record Sequence Number (RSN) of the database and the horizontal component used for the analyses, as well as information about the earthquake event and measuring station.

The accelerograms are assumed to correspond to the free-field motion at surface level. For the purpose of comparing the response of the different accelerograms, all results presented in this work are normalized per unit of maximum ground acceleration (acceleration units in $\mathrm{g}$ ). Fig. 2 displays the normalized acceleration spectra of the selected excitation signals. 


\begin{tabular}{cccccc}
\hline RSN & Component & Event Name & Year & Station Name & $V_{s, 30}(\mathrm{~m} / \mathrm{s})$ \\
\hline 56 & 130 & San Fernando & 1971 & Carbon Canyon Dam & 235 (Type D) \\
165 & 282 & Imperial Valley-06 & 1979 & Chihuahua & 242 (Type D) \\
338 & 0 & Coalinga-01 & 1983 & Parkfield - Fault Zone 14 & 246 (Type D) \\
722 & 270 & Superstition Hills-02 & 1987 & Kornbloom Road (temp) & $266($ Type D) \\
766 & 0 & Loma Prieta & 1989 & Gilroy Array \#2 & $271($ Type D) \\
931 & 180 & Big Bear-01 & 1992 & San Bernandino - E \& Hospitality & 297 (Type D) \\
1084 & 52 & Northridge-01 & 1994 & Sylmar - Converter Sta & 251 (Type D) \\
178 & 230 & Imperial Valley-06 & 1979 & El Centro Array \#3 & 163 (Type E) \\
334 & 0 & Coalinga-01 & 1983 & Parkfield - Fault Zone 1 & 178 (Type E) \\
718 & 90 & Superstition Hills-01 & 1987 & Imperial Valley Wildlife Liquefaction Array & 179 (Type E) \\
729 & 360 & Superstition Hills-02 & 1987 & Imperial Valley Wildlife Liquefaction Array & $179($ Type E) \\
759 & 90 & Loma Prieta & 1989 & Foster City - APEEL 1 & 116 (Type E) \\
962 & 180 & Northridge-01 & 1994 & Carson - Water St & 161 (Type E) \\
4100 & 360 & Parkfield-02_CA & 2004 & Parkfield - Cholame 2WA & $173($ Type E) \\
\hline
\end{tabular}

Table 1: Information about the accelerograms used as excitation in the analyses. Source: PEER Ground Motion Database [35]

Type D

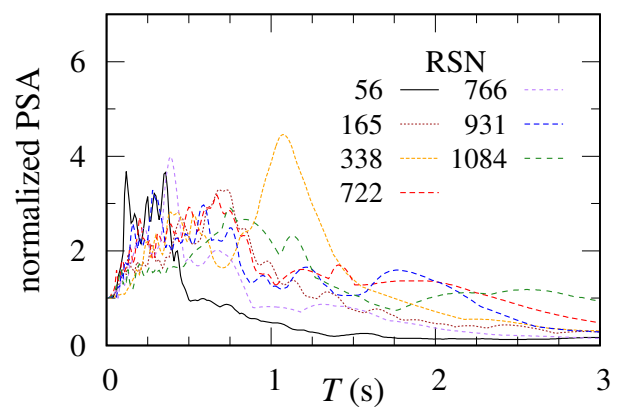

Type E

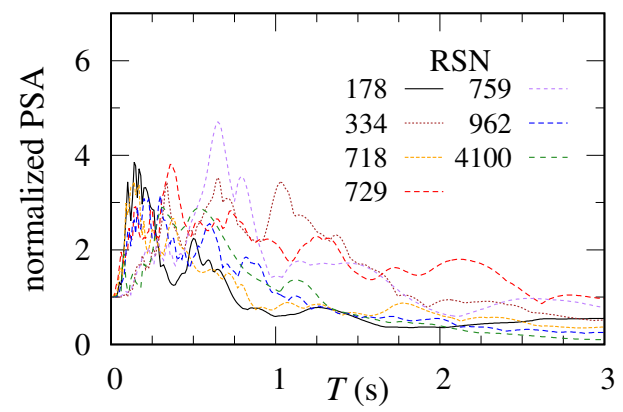

Figure 2: Normalized acceleration spectra of the selected accelerograms used as excitation.

\section{Methodology}

The foundation response is obtained through a time-harmonic three-dimensional numerical model previously developed by the authors [36, 37, 38]. Linear-elastic behaviour of soil and piles is assumed. The formulation is based on the reciprocity theorem in elastodynamics and the use of particular Green's functions for the layered half space [39]. Piles are treated as Timoshenko's beam elements and are included as load lines, in terms of soil-pile interaction tractions, in the soil formulation. Note that the Timoshenko's theory is used due to the general scope of the model, but it is not strictly necessary for the aspect ratios considered in the problem at hand. The soil and pile formulations are coupled together by imposing equilibrium and compatibility conditions, respectively, over these soil-pile interaction tractions and the displacements of the internal points of the soil and the beam element nodes located at the same coordinates (no sliding or gap between soil and pile is allowed). Pile groups can be considered by linking the motion of the pile heads through a rigid massless cap. Both fixed or hinged pile-cap union conditions can be considered by imposing or not the corresponding rotation restrictions. The pile cap is assumed as not in contact with the soil, so all the soil-foundation interaction is limited to the one produced along the pile shafts.

The seismic excitation is assumed to be generated by planar wave-fronts propagating through the layered media and is included in the soil formulation through the classical decomposition 


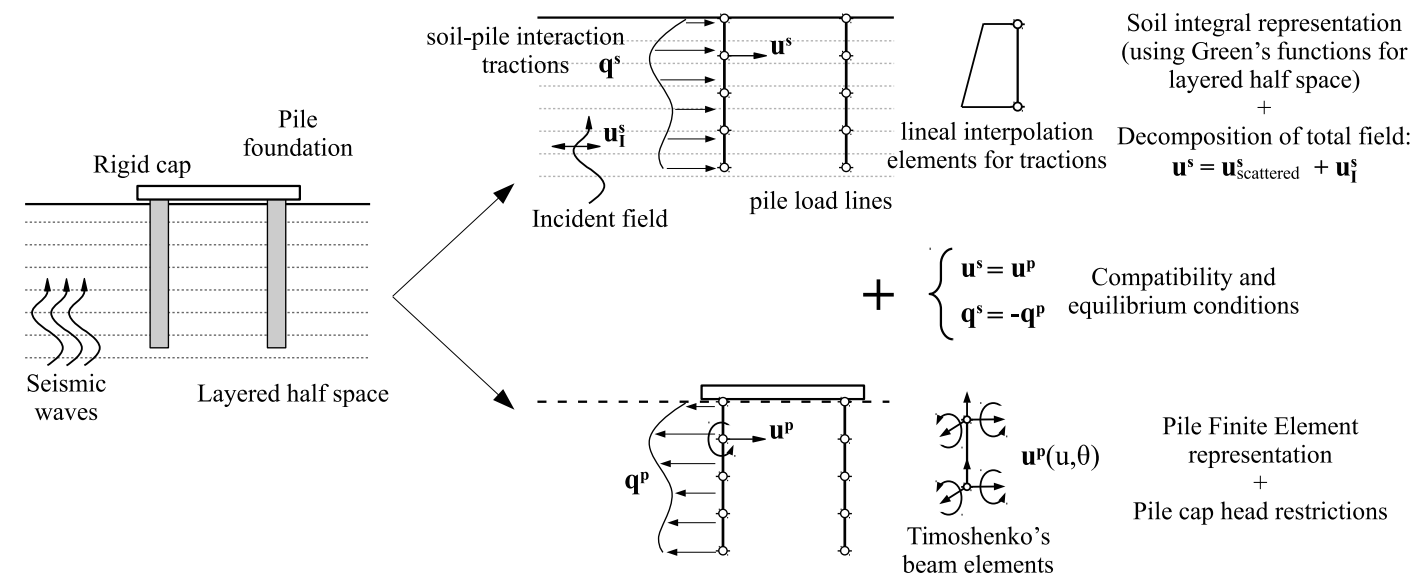

Figure 3: Sketch of the integral model used to compute the pile response.

of the total field into the incident field (produced by the body waves) plus the scattered field (produced by the presence of the piles) [40,41, 42]. Figure 3 shows a sketch of the model.

The main advantage of the model with respect to previous formulations employed by the authors, such as Boundary Elements [43] or coupled Boundary Elements - Finite Elements [41], is its capability to accurately reproduce the foundation response without the necessity of any discretization of the soil domain. This is obtained thanks to the use of the specific Green's functions for the layered half space that already satisfy the free-surface and layer interface boundary conditions [39]. The model has been validated against multi-domain Boundary Element codes (see, e.g. [38]) and results previously published in the literature (see, e.g. [36, 37]), satisfactorily showing the capability of the proposed tool to solve various dynamic problems involving soil-pile and pile-soil-pile interaction phenomena.

For the problem addressed in this work, the standard frequency-domain method [44] is employed to compute the time-evolution of the kinematic bending moments and its corresponding envelopes of maximum values. After computing the envelopes for each excitation signal, the response of each configuration is represented by the maximum value obtained at each point of the pile (regardless of the accelerogram to which it corresponds).

\section{Results}

The influence of the soil profile on the envelopes of kinematic bending moments of piles is clearly illustrated in Fig. 4. It presents the results obtained considering single piles with fixed-rotation at their heads and a pile length $L=50 \mathrm{~m}$. The results corresponding to the different types of soil profiles (homogeneous, linear and power-law) are plotted by using different colors, while the two studied ground types (D and E) are separated in two rows: top row for the harder type $\mathrm{D}$ and bottom row for the softer type E. The envelopes of several pile diameters are shown in the different columns. The maximum value among the results of the seven accelerogram is plotted in solid lines, while the variability due to the excitation signal is illustrated by the shaded area that represents, at each point of the pile shaft, the interval within the maximum and minimum values of the envelopes obtained among the seven accelerograms. As mentioned before, in order to compare the results corresponding to the different excitation signals, the envelopes of kinematic bending moments are presented normalized by the maximum ground acceleration. 

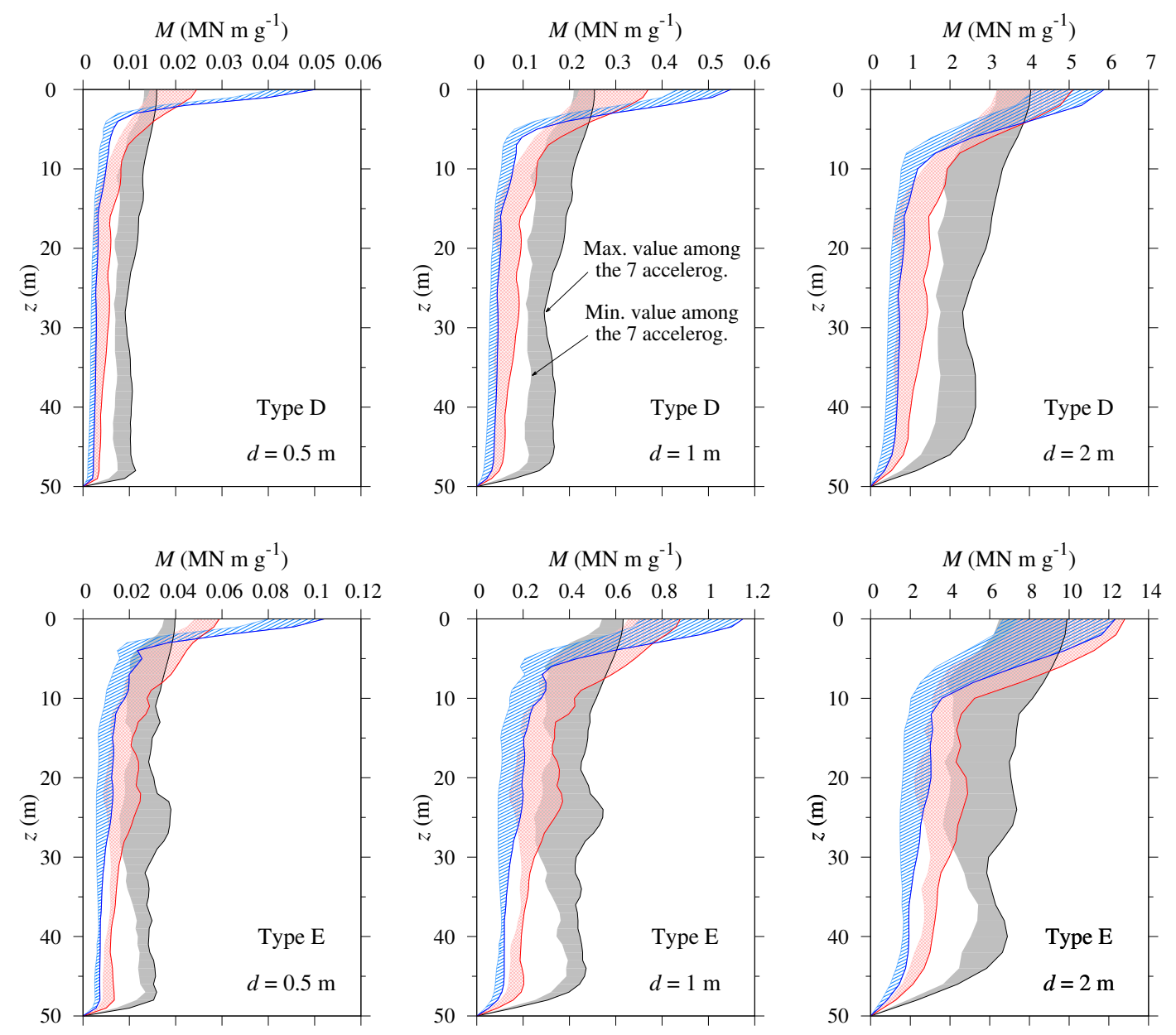

Figure 4: Envelopes of maximum kinematic bending moments for the homogeneous (black), linear (red) and power-law (blue) profiles. Maximum value of the seven accelerograms (lines) and area comprised between their minimum and maximum values. 
The results clearly manifest that, despite having identical average shear wave velocities, the envelopes obtained for the three profiles are significantly different. Two zones with distinct behaviour are distinguished. In the superficial layers, the variable profiles present larger bending moments than the homogeneous one. On the contrary, for deeper points (below 5-10 m depending on the configuration), the assumption of a homogeneous profile leads to the largest bending moments. The model of the power-law variation of the soil shear wave velocity presents the strongest contrasts in stiffness with depth and, at the same time, the softest soil near the surface together with the stiffest soil near the pile tip. For this reason $[6,9]$, this ground profile is the one that leads to the largest bending moments at pile head (over a $225 \%$ with respect to the ones for the homogeneous profile) and, at the same time, the smallest bending moments in the deepest portions of the piles (less than a $25 \%$ of the ones for the homogeneous soil). However, it is found that the point at which the different envelopes intersect with each other is closer to the free surface compared to the point at which their shear wave velocity profiles do. To understand this small gap, the behaviour of the incident field for the different profiles should be considered. In the variable profiles, the conservation of the energy that propagates through the soil makes the amplitude of the displacements of the incident field to decrease as the stiffness of the soil increases with depth. This amplitude reduction due to the characteristic of the variable profile can be seen, e.g. in the expressions obtained by Rovithis et al. [45] for the soil displacements, which are based on Bessel functions that intrinsically include this attenuation. The magnitude of the kinematic bending moment being related to the magnitude of the incident field, at depths in which the three profiles present similar stiffnesses, the effect of the incident field is large. At greater depths, as mentioned before, the influence of the stiffness differences is more important than the one of the incident field.

Fig. 4 also illustrates the well-known importance of using various accelerograms when studying the maximum temporal response of piles. For clarity's sake, Fig. 5 presents the relative difference between the maximum and minimum envelope obtained among the seven accelerograms. The results show that, in general terms, the three considered profile types lead to similar relative variations between the maximum and minimum response (approximately around 0.4).

In order to further analyse the effects of the soil profile and study the aforementioned phenomena in more detail, Figs. 6 and 7 present a synthesis of the results obtained at the pile head and shaft, respectively. In these figures, the influence of the pile diameter on the maximum kinematic bending moments is investigated. For the purpose of removing the known dependence of the bending moment on the pile cross-section moment of inertia, results are presented in terms of the pile curvature defined as:

$$
(1 / R)_{\text {pile }}=\frac{M}{E I} \propto \frac{M}{d^{4}}
$$

First, Fig. 6 presents the maximum pile curvature obtained at the pile head with restricted rotation. The behaviour obtained for the two ground types is the same. The pile curvature at the pile head is practically independent of the pile diameter in the homogeneous media. Furthermore, the use of this profile significantly underestimates the head bending moments that are obtained for the two variable profiles with the same average velocity. For the linear profile, also the influence of the pile diameter is negligible on the pile curvature, although a small monotonic decrease of its value is seen as the pile dimension increases. On the other hand, for the power-law profile the pile curvature at the pile head is significantly influenced by the pile diameter, exponentially reducing its value as the diameter increases. This agrees with the findings of Di Laora et al. [46] about the reduction of the effect of pile diameter in the head maximum kinematic bending moment for non-homogeneous profiles, i.e. the exponent $n$ in the relation $M \propto d^{n}$ is lower than 4 for the variables profiles. In fact, from the results it is found 

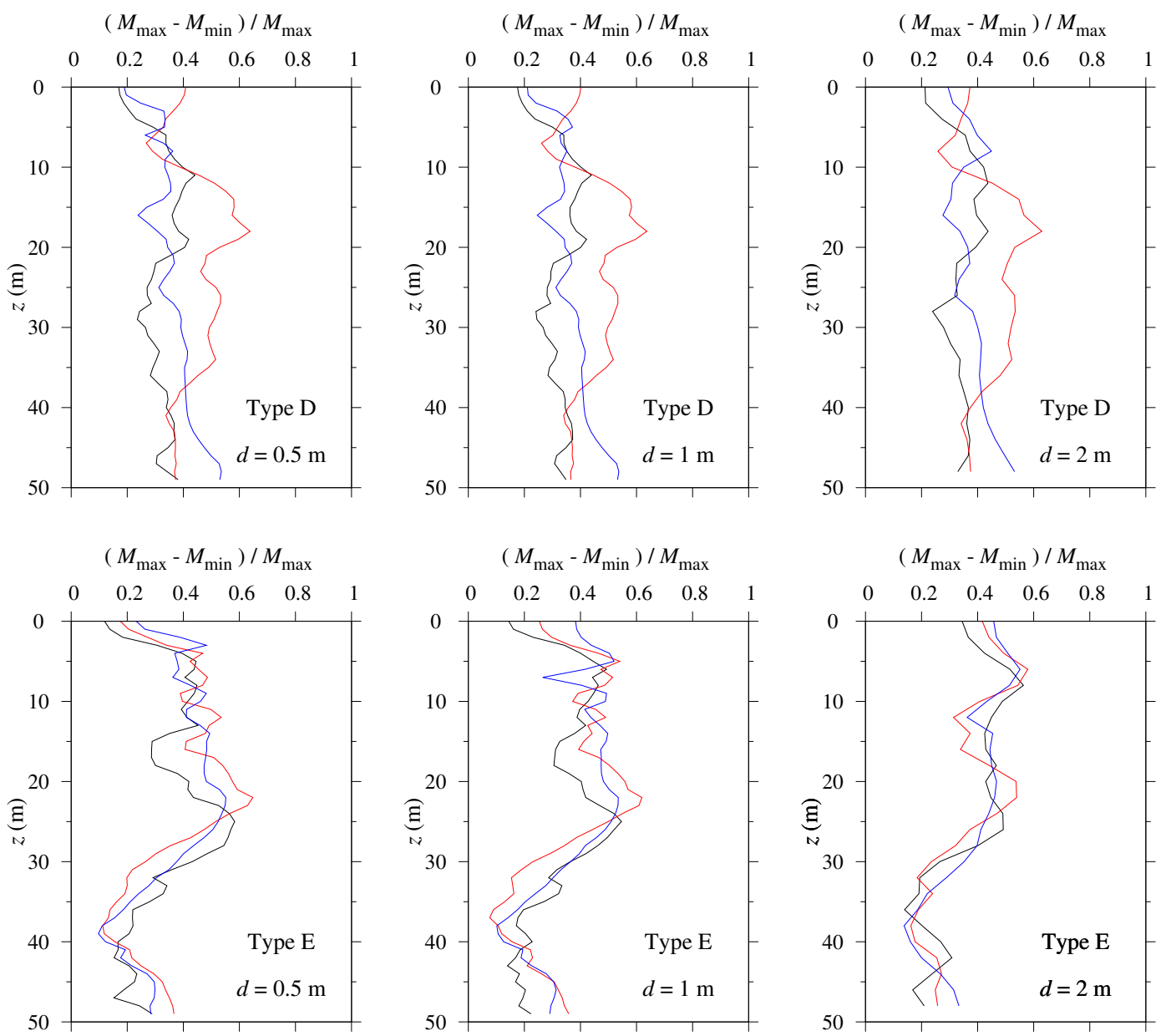

Figure 5: Relative difference between the maximum and minimum values of the envelopes of maximum kinematic bending moments among the seven accelerograms. Results for the homogeneous (black), linear (red) and power-law (blue) profiles.

Type D

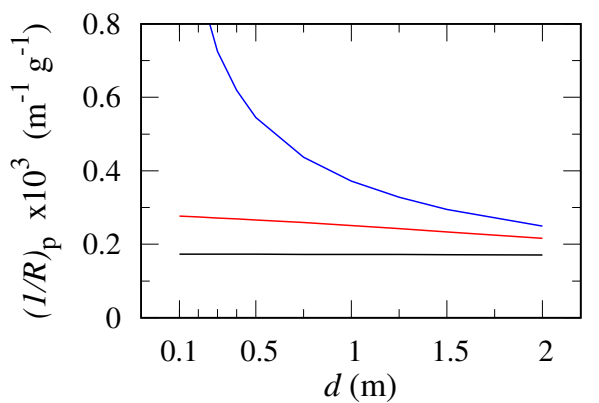

Type E

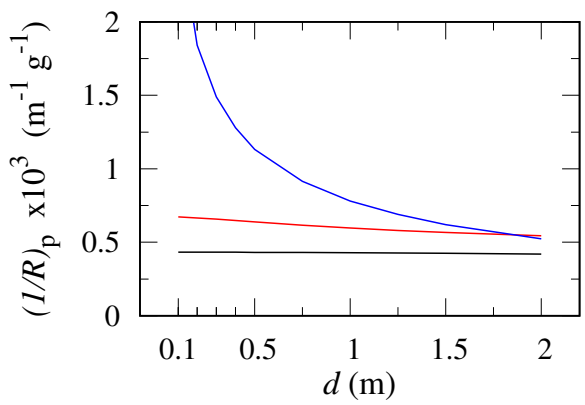

Figure 6: Maximum pile curvature at the pile head obtained for the most unfavorable accelerogram. Comparison between the homogeneous (black), linear (red) and power-law (blue) profiles. 
Type D

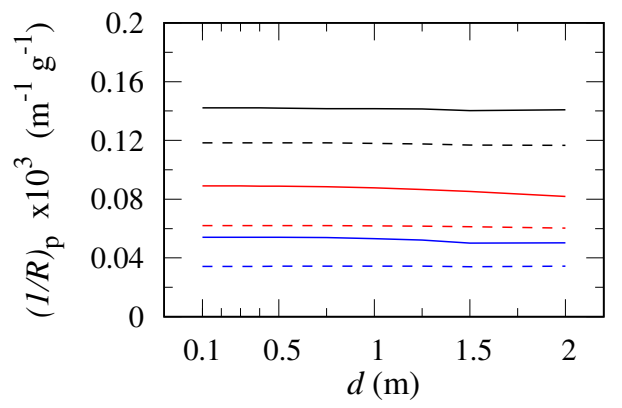

Type E

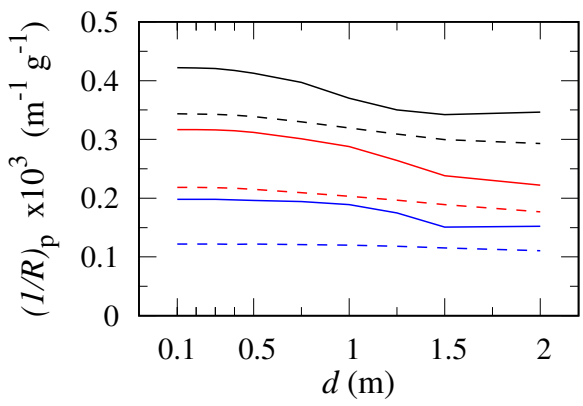

Figure 7: Summary of the pile maximum kinematic bending moments between depths from 10 to $40 \mathrm{~m}$. Maximum (solid lines) and shaft-average (dashed lines) values of the pile curvature obtained for the most unfavorable accelerogram. Comparison between the homogeneous (black), linear (red) and power-law (blue) profiles.

that $n=4$ for homogeneous media, $n=3.9$ for the linear profile and $n=3.45$ for the power-law one. As it will be shown in Section 4.1, the response at any point of the pile is mainly influenced by the characteristics of its near surroundings. When the pile diameter increases, the depth of soil that affects the head bending moment also increases and, therefore, the corresponding average soil stiffness augments. This explains the decrease in the pile-head bending moment with the diameter of the variable soils, which is much more evident in the power-law profile due to its stronger dependence with depth. Thus, depending on the diameter, the equivalent homogeneous soil with the same average shear wave velocity produces maximum head bending moments that are 1.5-1.3 times lower than the ones obtained for the linear profile; or between 3 and 1.25 times lower with respect to the power-law profile.

On the other hand, Fig. 7 shows the behaviour that is obtained along the points of the pile located between 10 and $40 \mathrm{~m}$. This portion of the pile is defined as it is not influenced by the boundary conditions imposed at the pile head or tip (as will be shown in Section 4.1) and, therefore, represents the expected response of the pile shaft due to the seismic excitation. This response is characterized through two magnitudes: the maximum value obtained along the considered length (solid lines), and the shaft-average value obtained along this portion of the pile shaft (dashed lines). The first is chosen as the most critical value that the pile section should stand, while the second indicates the general trend of the bending moments along the pile shaft. As commented before, and contrary to what occurs for the pile head, the homogeneous profile overestimates the maximum bending moments of the pile shaft. The equivalent homogeneous soil produces pile curvatures that are over 1.6 times and 2.5 times the ones produced by the linear and power-law profiles, respectively. Nevertheless, the dependence of the pile curvature with the pile diameter is the same for the three profiles. For ground type D, both the value of the maximum and average pile curvature along the pile shaft are almost independent of the pile dimensions. For ground type E, the pile curvature starts to decrease with increasing pile diameter, its maximum value being noticeably higher for thinner piles. This behaviour is a known effect in the kinematic soil-pile interaction (see, e.g. [11, 13]): when the pile-soil stiffness contrast is low or large wavelengths in the soil are involved, pile response is mainly determined by soil. But when the pile-soil stiffness ratio or the frequency increases, the pile can not follow the soil movement and its response is reduced. 


\subsection{Influence of boundary conditions}

The analysis of the different single pile configurations shows that, for piles of the same diameter, the kinematic bending moments practically coincide along the shaft, presenting only differences at the surroundings of the pile head and tip. The comparison of the envelopes of maximum kinematic bending moments of the studied single pile configurations is shown in Fig. 8. This figure superimposes the results obtained for piles of length $L=10,20,30,40$ and $50 \mathrm{~m}$ assuming both the free and fixed-rotation head conditions. Only the results for ground type $\mathrm{E}$ are presented to illustrate this phenomenon, as the same trend is observed for ground type D.

From the results presented in Fig. 8, it is found that, with the exception of the points located near the pile extremes (i.e., head or tip), the envelopes of kinematic bending moments are independent of the pile length and head condition. In other words, the envelopes of maximum kinematic bending moments are independent of pile length provided that the pile is slender. This is the reason why the configuration of the longest pile $(L=50 \mathrm{~m})$ with fixed-rotation head condition has been the only one studied in the previous section.

\begin{tabular}{cccc}
\hline Ground type & Homogeneous & Linear & Power-law \\
\hline $\mathrm{D}$ & $4.2 d$ & $5.0 d$ & $6.0 d$ \\
$\mathrm{E}$ & $5.2 d$ & $5.8 d$ & $6.4 d$ \\
\hline
\end{tabular}

Table 2: Depth of influence of the pile head boundary condition for the studied profiles.

The length of the influence zone of the boundary conditions is found to be proportional to the pile diameter and, to a lower extent, to the ground type and profile (i.e., stiffness of the surrounding soil). The magnitude of the length of the influence zone of the head condition has been estimated. For this purpose, the point at which the two envelopes corresponding to the free and fixed-rotation conditions intersect is calculated. The average value obtained from the seven excitation signals and all studied configurations is expressed in terms of the pile diameter in Table 2. As expected, the dimension of the influence zone of the head conditions decreases when the soil stiffness increases. This effect can be easily explained with the aid of the expressions of the kinematic active length proposed by Di Laora and Rovithis [16] and Karatzia and Mylonakis [17] based on a Winkler model. In fact, the concepts of the kinematic active length and influence zone are equivalent (the first is the length from which increasing the pile length does not alter the pile-head response; while the second is the length that is affected by the pile-head conditions). For example, using the expressions of [17] considering $\chi=2.5, \delta=2$ and $d=1 \mathrm{~m}$, the active lengths for the profiles of type $\mathrm{D}$ are $4.4 d$ (homogeneous), $4.9 d$ (linear) and $6.0 d$ (power-law), which agree quite well with the results presented in Table 2.

The influence zones of the pile head condition according to the values presented in Table 2 are illustrated in Fig. 8 with a grey shading. These areas accurately indicate the portion of the pile in which the envelopes corresponding to the free and fixed-rotation head conditions diverge. Despite the necessity of a more exhaustive analysis, it is expected that the influence zones indicated by Table 2 correspond to the portion of the pile more affected by the inertial loads imposed by the supported structure. For the rest of the pile shaft, the maximum bending moments due to a seismic excitation are expected to be similar to the ones presented in this study regardless of the existence of superstructure.

On the other hand, the orange shadings apply these same influence zones to each pile tip condition. As expected, for the homogeneous profile the values presented in Table 2 can be also used to estimate the point at which the results corresponding to piles of different length diverge due to the floating tip condition. However, these values overestimate the influence zone of the pile tip condition in the case of the variable profiles, especially for the power-law evolution. This 

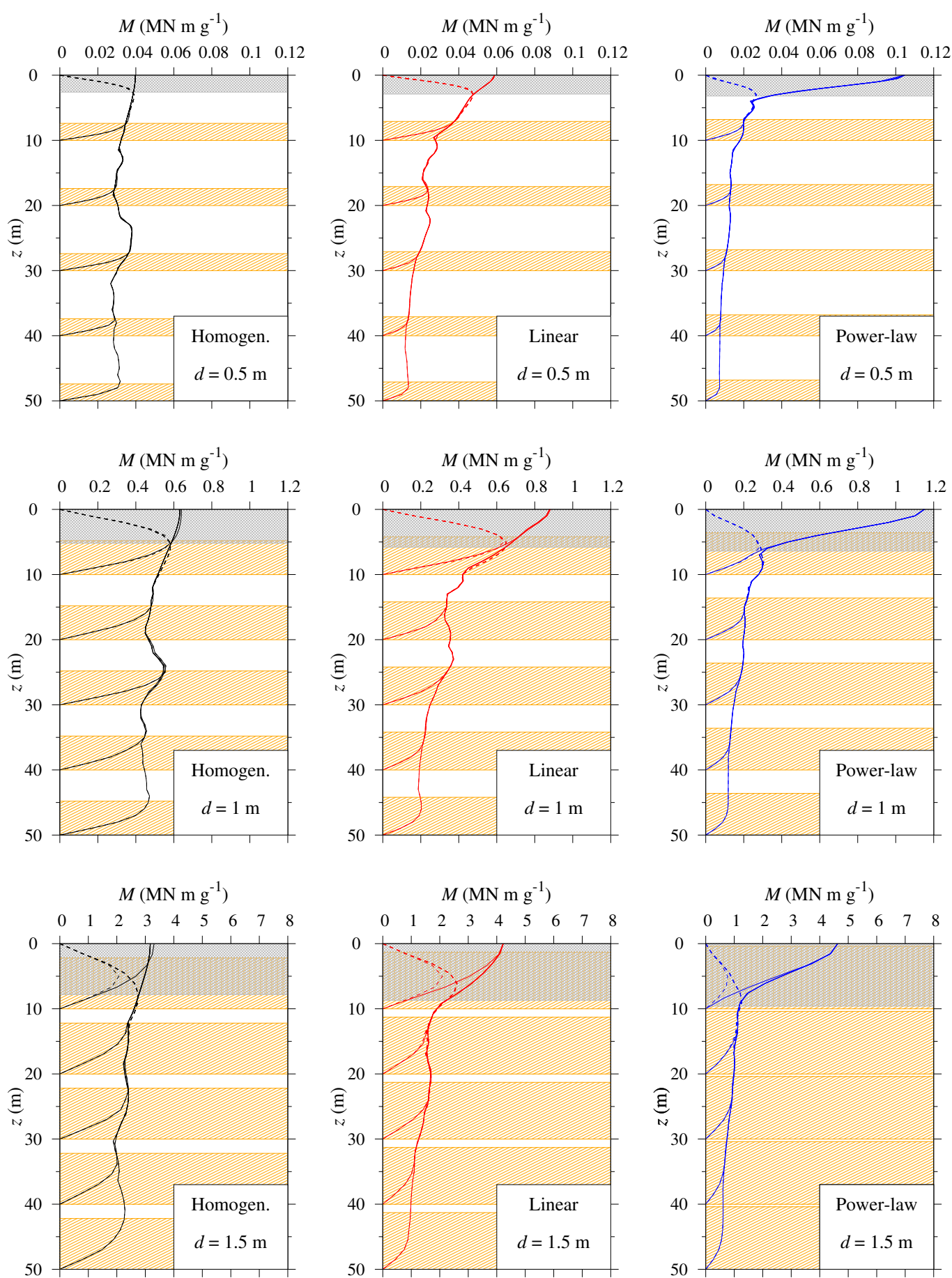

Figure 8: Zones of influence of the head (grey area) and tip (orange area) boundary conditions on the envelopes of maximum kinematic bending moments (Table 2). Maximum value of the seven accelerograms for fixed-rotation (solid lines) and free (dashed lines) head conditions. Ground type E. 
happens because the ranges presented in Table 2 are computed from the results at the pile head, corresponding to softer soil average properties than the ones found at the pile tip (the stiffer the soil, the shorter the influence zone of the boundary conditions).

Finally, the special case of the pile configurations with diameter $d=1.5 \mathrm{~m}$ and length $L=10$ $\mathrm{m}$ should be highlighted. For these foundations, almost the whole pile is affected by both the head and tip boundary conditions. Therefore, their envelopes of maximum bending moments significantly differ from the ones of the rest of configurations.

\subsection{Influence of the pile-soil-pile interaction effects}

In the previous sections, the single pile problem has been addressed. In this section, it is verified that the effects of the soil profile already commented for the single pile can be applied for group configurations. Furthermore, the magnitude of the pile-soil-pile interaction effects on the envelopes of kinematic bending moments is analysed. For these purposes, foundations formed by $2 \times 2,3 \times 3$ and $4 \times 4$ pile groups are investigated. Firstly, the study presented in this section considers the separation distance $s / d=2$ as the limit scenario in which the pile-soil-pile interaction effects have the largest impact. A fixed union between the piles and the rigid cap is assumed, while no restriction is imposed to the group rotation.

Fig. 9 compares the envelopes of maximum kinematic bending moments of the group configurations with respect to the ones already presented for the single pile in Fig. 4 . For the $3 \times 3$ and $4 \times 4$ groups, only the response of the central piles are shown. However, the variations between the envelopes of the different piles in these groups are negligible. The results presented in Fig. 9 confirm that the response of the grouped piles are virtually coincident with those of the single pile. The pile-soil-pile interaction becomes more evident for soft soils and large diameters. In general terms, the pile-soil-pile interaction produces a slight reduction of the kinematic bending moments (up to a $20 \%$ for large pile diameters), the results of the single pile being the upper bound in all scenarios. Note that, despite not having a fixed-rotation condition, the large vertical stiffness of the piles in the group significantly limits the foundation rotation. As a result, the group response converge to the one of the single pile with fixed-rotation head conditions.

Giving a closer look to the head response, Fig. 10 shows the pile curvature obtained for the different configurations. As mentioned before, the effects of the soil profile, as well as the influence of the diameter of the pile, are the same regardless of the pile being single or part of a group. For the group configurations, the maximum head curvature is reduced with respect to the fixed-rotation single pile due to different reasons: the pile-soil-pile interaction effects and the relaxation of the rotation restrain condition. As the number of piles in the configuration increases and, in consequence, the vertical stiffness of the additional piles restrict more group rotation, the maximum head bending moment sightly increases.

On the other hand, Fig. 11 presents the comparison of the results corresponding to the part of the pile shaft between 10 and $40 \mathrm{~m}$. For brevity's sake, only the shaft-average values are shown, but similar effects are observed when studying the maximum value produced along this portion of the pile. Again, a small reduction of the pile curvature due to the presence of near piles is found. The reduction is only noticeable for the softest ground type (Type E). In this case, the differences with respect to the single pile configuration augment with the number of piles in the group.

Finally, the influence of the pile separation distance $s / d$ on the envelopes of kinematic bending moments is also studied. As expected, when its value increases, the influence of the pile-soil-pile interaction decreases, converging the results of the piles in the groups to the ones of the single pile. For illustration purposes, Fig. 12 shows the envelopes of maximum kinematic bending moments obtained for several $2 \times 2$ configurations with $s / d=2,4,6,8$ and 10 , and compares 

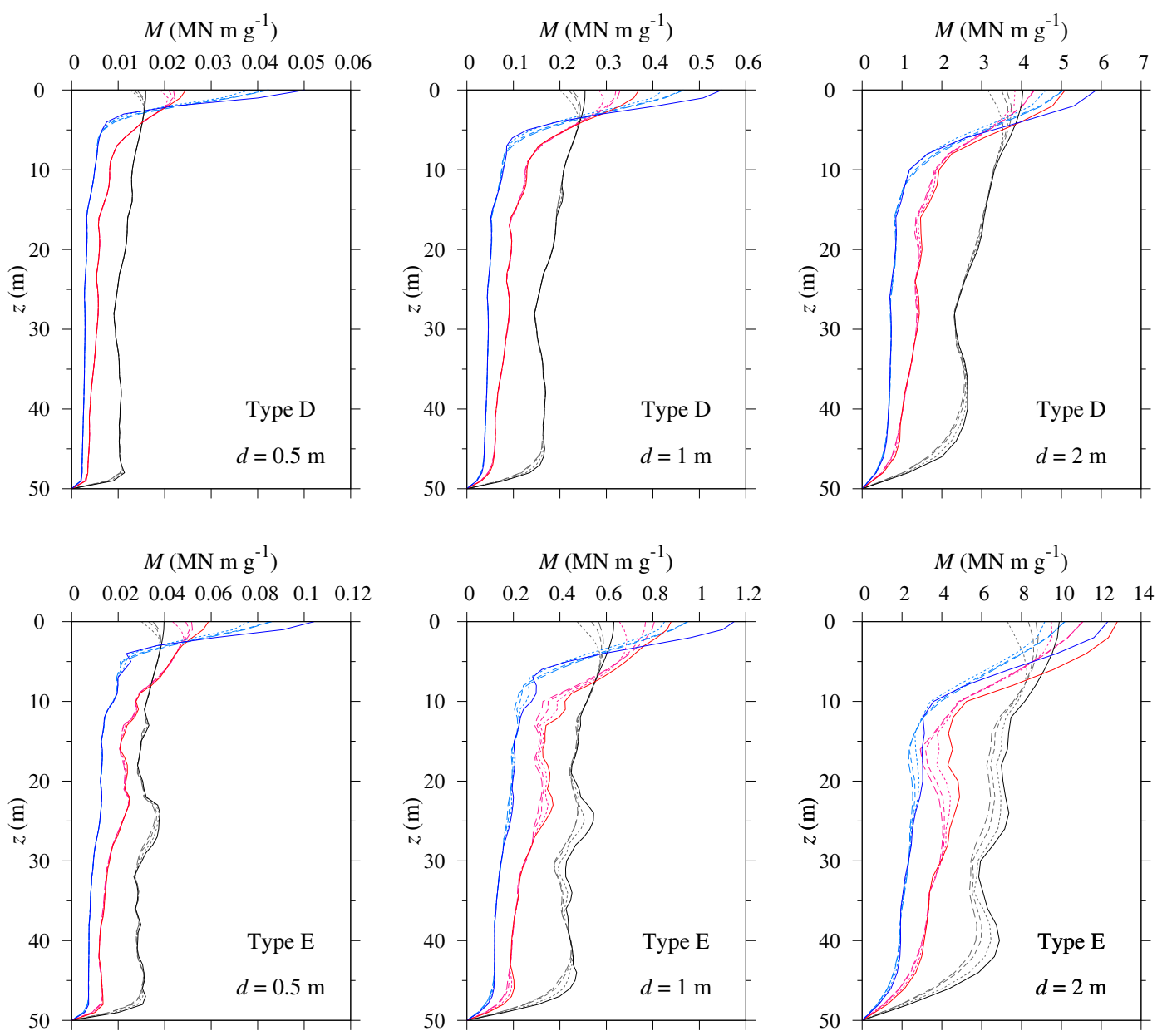

Figure 9: Influence of group effects on the envelopes of maximum kinematic bending moments for the homogeneous (black), linear (red) and power-law (blue) profiles. Results for single pile (solid), and $2 \times 2$ (short dash), $3 \times 3$ (medium dash) and $4 \times 4$ (large dash) pile groups with $s / d=2$. Maximum value of the seven accelerograms.

Type D

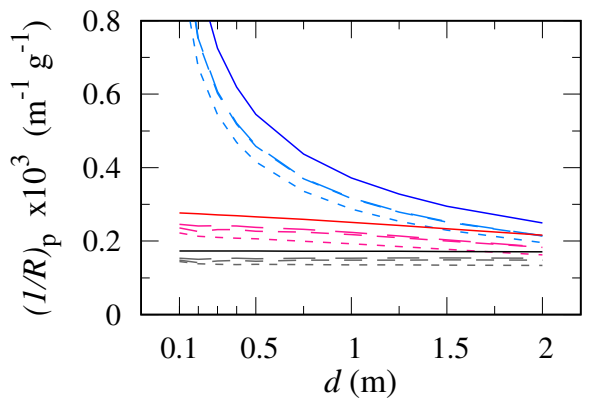

Type E

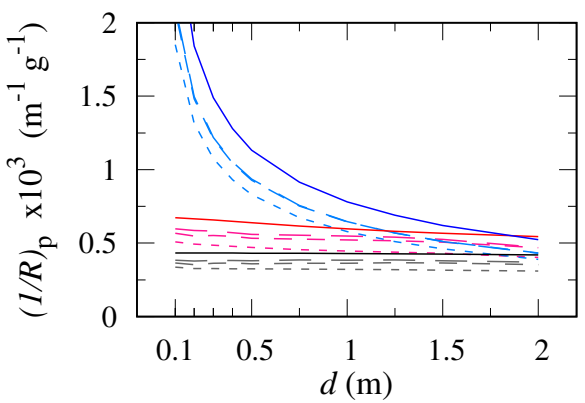

Figure 10: Influence of the group effects on the maximum pile curvature at the pile head. Comparison between the homogeneous (black), linear (red) and power-law (blue) profiles. Results for single pile (solid), and $2 \times 2$ (short dash), $3 \times 3$ (medium dash) and $4 \times 4$ (large dash) pile groups. 
Type D

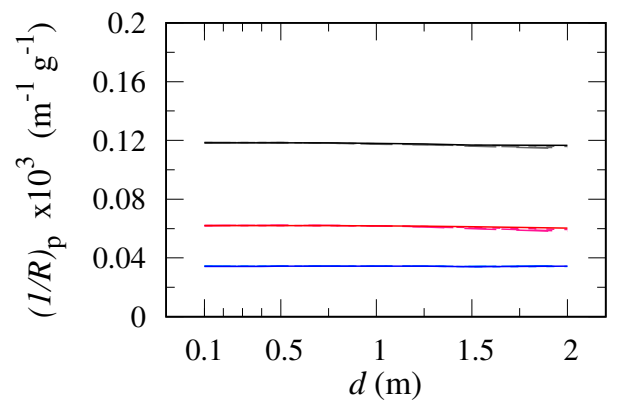

Type E

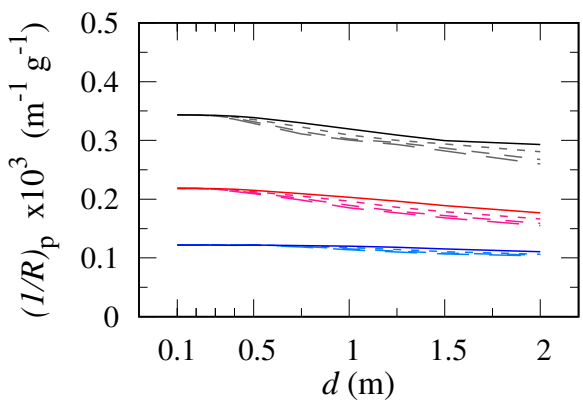

Figure 11: Influence of the group effects on the shaft-average (from 10 to $40 \mathrm{~m}$ ) value of the maximum pile curvature. Comparison between the homogeneous (black), linear (red) and powerlaw (blue) profiles. Results for single pile (solid), and $2 \times 2$ (short dash), $3 \times 3$ (medium dash) and $4 \times 4$ (large dash) pile groups.

them to the ones obtained by the single pile. Only results for the ground type E are presented as in this ground type the pile-soil-pile interaction effects are more noticeable than for ground type D. It is found that significant differences among the different configurations can just be seen at the head of the piles (due to the relaxation of the fixed-rotation condition, which is more evident for smaller foundations as they present larger head rotations), but the group envelopes rapidly converge to the one of the single pile as the separation distance increases.

\section{Conclusions}

The importance of the definition of the soil profile in the estimation of the maximum kinematic bending moments of pile foundations is studied. Several soil profiles based on real boreholes together with typical pile dimensions are considered for the analyses. The foundation response is computed through a time-harmonic integral model based on the reciprocity theorem in elastodynamics and the use of specific Green's functions for layered soils. In this formulation, piles are treated as beam elements. Envelopes of maximum bending moments are computed through the standard frequency-domain method and the use of accelerograms corresponding to actual events measured in stations located at soils with similar characteristics. Note that due to the characteristics of the considered model, all the conclusions are only valid within the linear elasticity framework. However, previous works $[8,46]$ remarked that soil material nonlinearity effects are negligible in kinematic soil-pile interaction (although they are dominant in the free field response), so the results of this study could still be applicable under such conditions.

The obtained results confirm that the evolution-with-depth of the soil profile plays a major role on the maximum kinematic bending moments of pile foundations. The studied profiles, despite having the same average shear wave velocity, produce significantly different pile responses. Thus, the use of an equivalent homogeneous soil (in terms of $V_{s, 30}$ ) is not recommended for the estimation of the kinematic bending moments of piles embedded in variable profiles. The effects of this assumption are different depending on the part of interest of the pile: at the pile head, the homogeneous profile underestimates the maximum bending moments that are produced for the variable media, while along the pile shaft (approx. from depths greater than 5-8 $\mathrm{m}$ ) the opposite trend is found. Therefore, for an accurate analysis of kinematic bending moments along the whole pile, the use of soil-foundation models that take into account the inhomogeneity of the soil profile with depth is recommended. 

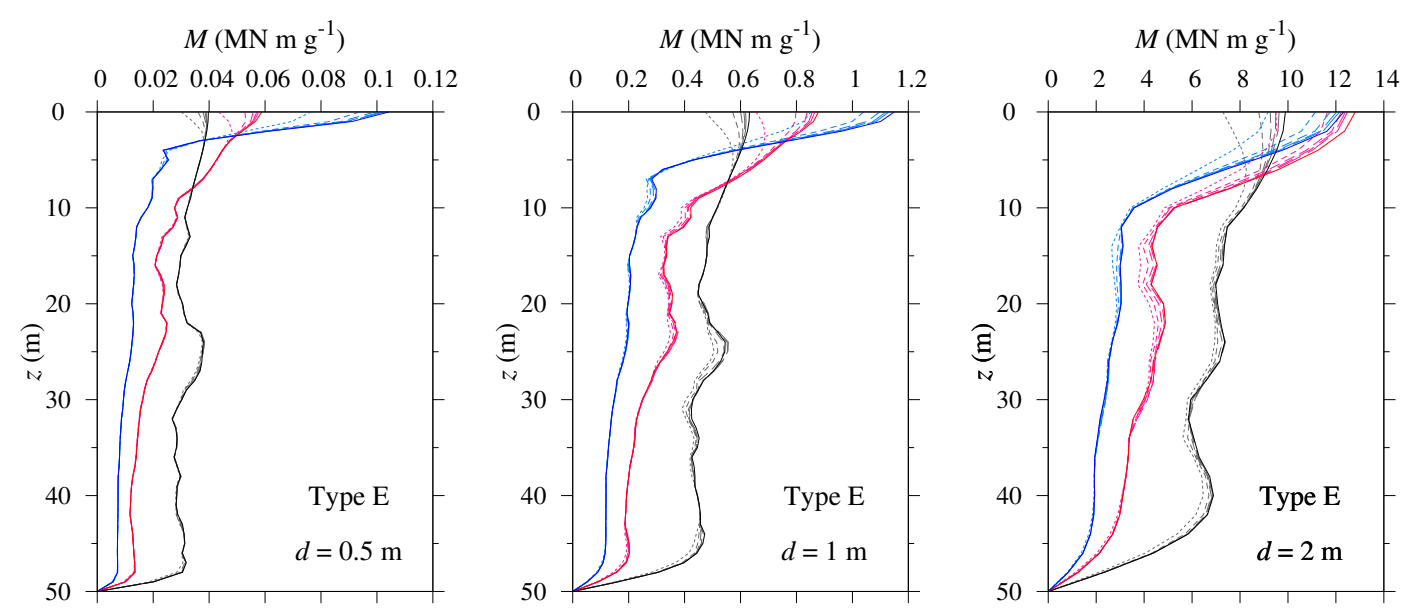

Figure 12: Influence of group effects on the envelopes of maximum kinematic bending moments for the homogeneous (black), linear (red) and power-law (blue) profiles. Results for single pile (solid), and $2 \times 2$ pile groups with $s / d=2,4,6,8,10$ (dash size proportional to separation distance). Maximum value of the seven accelerograms.

The effects of the soil profile are identical for single pile configurations or pile groups. Furthermore, only in foundations with close piles with large diameters embedded in soft soils the pile-soil-pile interaction effects have some impact on the maximum kinematic bending moments. In this situation, these interaction effects slightly reduces the magnitude of the pile maximum response.

\section{Acknowledgments}

This work was supported by the Subdirección General de Proyectos de Investigación of the Ministerio de Economía, Industria y Competitividad (MINECO) and Agencia Estatal de Investigación (AEI) of Spain and FEDER through research project BIA2017-88770-R. G.M. Álamo is a postdoctoral fellow of the ULPGC Postdoctoral Programme. The authors would also like to thank the anonymous Reviewers whose comments and suggestions have helped to improve the manuscript.

\section{References}

[1] H. Mizuno. Pile damage during earthquake in Japan (1923-1983). In T. Nogami, editor, Dynamic Response of Pile Foundations - Experiment, Analysis and Observation, pages 5378. Geotechnical Special Publication ASCE, 1987.

[2] T. Tazoh, K. Shimizu, and T. Wakahara. Seismic observations and analysis of grouped piles. In T. Nogami, editor, Dynamic Response of Pile Foundations - Experiment, Analysis and Observation, pages 1-20. Geotechnical Special Publication ASCE, 1987.

[3] S. Nikolaou, George Mylonakis, G. Gazetas, and T. Tazoh. Kinematic pile bending during earthquakes: analysis and field measurements. Geotechnique, 51(5):425-440, 2001. 
[4] Mahmoud N Hussien, Tetsuo Tobita, Susumu Iai, and Mourad Karray. Soil-pile-structure kinematic and inertial interaction observed in geotechnical centrifuge experiments. Soil Dyn Earthq Eng, 89:75-84, 2016.

[5] Francesca Dezi, Sandro Carbonari, and Graziano Leoni. A model for the 3D kinematic interaction analysis of pile groups in layered soils. Earthq Eng Struct Dyn, 38:1281-1305, 2009.

[6] Francesca Dezi, Sandro Carbonari, and Graziano Leoni. Static equivalent method for the kinematic interaction analysis of single piles. Soil Dyn Earthq Eng, 30(8):679-690, 2010.

[7] Stefania Sica, George Mylonakis, and Armando Lucio Simonelli. Strain effects on kinematic pile bending in layered soil. Soil Dyn Earthq Eng, 49:231-242, 2013.

[8] Mario Martinelli, Alberto Burghignoli, and Luigi Callisto. Dynamic response of a pile embedded into a layered soil. Soil Dyn Earthq Eng, 87:16-28, 2016.

[9] G. Mylonakis. Simplified model for seismic pile bending at soil layer interfaces. Soils Found, 41(4):47-58, 2001.

[10] Rosa Maria Stefania Maiorano, Luca de Sanctis, Stefano Aversa, and Alessandro Mandolini. Kinematic response analysis of piled foundations under seismic excitation. Can Geotech J, 46(5):571-584, 2009.

[11] Luca de Sanctis, Rosa M S Maiorano, and Stefano Aversa. A method for assessing kinematic bending moments at the pile head. Earthq Eng Struct Dyn, 39(10):1133-1154, 2010.

[12] Stefania Sica, George Mylonakis, and Armando Lucio Simonelli. Transient kinematic pile bending in two-layer soil. Soil Dyn Earthq Eng, 31(7):891-905, 2011.

[13] Raffaele Di Laora, George Mylonakis, and Alessandro Mandolini. Pile-head kinematic bending in layered soil. Earthq Eng Struct Dyn, 42(3):319-337, 2013.

[14] Wenhai Ke, Qijian Liu, and Chao Zhang. Kinematic bending of single piles in layered soil. Acta Geotech, 14:101 - 110, 2019.

[15] Francesca Dezi and Harry Poulos. Kinematic bending moments in square pile groups. Int $J$ Geomech, 17(3):04016066, 2017.

[16] Raffaele Di Laora and Emmanouil Rovithis. Kinematic bending of fixed-head piles in nonhomogeneous soil. J Geotech Geoenviron Eng, 141(4):04014126, 2015.

[17] Xenia Karatzia and George Mylonakis. Discussion of kinematic bending of fixed-head piles in nonhomogeneous soil by raffaele di laora and emmanouil rovithis. J Geotech Geoenviron Eng, 142(2):07015042, 2016.

[18] Michele Mucciacciaro and Stefania Sica. Nonlinear soil and pile behaviour on kinematic bending response of flexible piles. Soil Dyn Earthq Eng, 107:195 - 213, 2018.

[19] Roger D. Borcherdt and Gary Glassmoyer. On the characteristics of local geology and their influence on ground motions generated by the Loma Prieta earthquake in the San Francisco Bay region, California. Bull Seismol Soc Amer, 82(2):603-641, 1992.

[20] Roger D. Borcherdt. Estimates of site-dependent response spectra for design (methodology and justification). Earthq Spectra, 10(4):617-653, 1994. 
[21] European Committee for Standardization. Eurocode 8: Design of structures for earthquake resistance. Part 5: Foundations, Retaining Structures and Geotechnical Aspects. Brussels, 2004.

[22] American Society of Civil Engineers (ASCE). Minimum Design Loads for Buildings and Other Structures. ASCE/SEI 7-05. Reston, VA, 2006.

[23] Silvia Castellaro, Francesco Mulargia, and Piermaria Luigi Rossi. Vs30: proxy for seismic amplification? Seismol Res Lett, 79(4):540-543, 2008.

[24] Vincent W. Lee and Mihailo D. Trifunac. Should average shear-wave velocity in the top 30m of soil be used to describe seismic amplification? Soil Dyn Earthq Eng, 30(11):1250 $1258,2010$.

[25] Ramón Verdugo. Seismic site classification. Soil Dyn Earthq Eng, 124:317 - 329, 2019.

[26] Domenico Di Giacomo, Maria Rosaria Gallipoli, Marco Mucciarelli, Stefano Parolai, and Sandra M. Richwalski. Analysis and modeling of HVSR in the presence of a velocity inversion: the case of Venosa, Italy. Bull Seismol Soc Amer, 95(6):2364, 2005.

[27] Jonathan P. Stewart, Andrew H. Liu, and Yoojoong Choi. Amplification factors for spectral acceleration in tectonically active regions. Bull Seismol Soc Amer, 93(1):332, 2003.

[28] Maria Rosaria Gallipoli and Marco Mucciarelli. Comparison of site classification from vs30, vs10, and hvsr in Italy. Bull Seismol Soc Amer, 99(1):340-351, 2009.

[29] Cadet Héloïse, Pierre-Yves Bard, Anne-Marie Duval, and Etienne Bertrand. Site effect assessment using KiK-net data: part 2-site amplification prediction equation based on f0 and Vsz. Bull Earthq Eng, 10(2):451-489, 2012.

[30] Kyriazis Pitilakis, Evi Riga, and Anastasios Anastasiadis. New code site classification, amplification factors and normalized response spectra based on a worldwide ground-motion database. Bull Earthq Eng, 11(4):925-966, 2013.

[31] L. Luzi, R. Puglia, F. Pacor, M. R. Gallipoli, D. Bindi, and M. Mucciarelli. Proposal for a soil classification based on parameters alternative or complementary to Vs,30. Bull Earthq Eng, 9(6):1877-1898, 2011.

[32] Yoshikazu Shingaki, Hiroyuki Goto, and Sumio Sawada. Evaluation performance for site amplification factors: S-wave impedance vs. Vs30. Soils Found, 58(4):911 - 927, 2018.

[33] Stefano Stacul and Nunziante Squeglia. Simplified assessment of pile-head kinematic demand in layered soil. Soil Dyn Earthq Eng, 130:105975, 2020.

[34] S Wang and H Wang. Site-dependent shear-wave velocity equations versus depth in California and Japan. Soil Dyn Earthq Eng, 88:8-14, 2016.

[35] Pacific Earthquake Engineering Research Center (PEER). NGA-West2 Ground Motion Database. http://ngawest2.berkeley.edu/ [Accessed on Apr 2019].

[36] G M Álamo, A E Martínez-Castro, L A Padrón, J J Aznárez, R Gallego, and O Maeso. Efficient numerical model for the computation of impedance functions of inclined pile groups in layered soils. Eng Struct, 126:379-390, 2016. 
[37] G M Álamo, J J Aznárez, L A Padrón, A E Martínez-Castro, and O Maeso. Importance of using accurate soil profiles for the estimation of pile kinematic input factors. $J$ Geotech Geoenviron Eng, 145(8):04019035, 2019.

[38] Guillermo M. Álamo, Jacob D.R. Bordón, Juan J. Aznárez, and Geert Lombaert. The effectiveness of a pile barrier for vibration transmission in a soil stratum over a rigid bedrock. Comput Geotech, 110:274 - 286, 2019.

[39] R Y S Pak and B B Guzina. Three-dimensional Green's functions for a multilayered halfspace in displacement potentials. J Eng Mech, 128(4):449-461, 2002.

[40] J Domínguez. Boundary elements in dynamics. Computational Mechanics Publications \& Elsevier Applied Science, Southampton, NY, 1993.

[41] L A Padrón, J J Aznárez, and O Maeso. Dynamic analysis of piled foundations in stratified soils by a BEM-FEM model. Soil Dyn Earthq Eng, 28(5):333-346, 2008.

[42] Chao Zhang, Peng Deng, and Wenhai Ke. Kinematic response of rectangular piles under s waves. Comput Geotech, 102:229 - 237, 2018.

[43] O. Maeso, J. J. Aznárez, and F. García. Dynamic impedances of piles and groups of piles in saturated soils. Comput Struct, 83:769-782, 2005.

[44] A K Chopra. Dynamic of structures. Theory and applications to earthquake engineering. NJ: Prentice-Hall, 2001.

[45] E. N. Rovithis, H. Parashakis, and G. E. Mylonakis. 1d harmonic response of layered inhomogeneous soil: Analytical investigation. Soil Dyn Earthq Eng, 31(7):879 - 890, 2011.

[46] Raffaele Di Laora, George Mylonakis, and Alessandro Mandolini. Size limitations for piles in seismic regions. Earthq Spectra, 33(2):729-756, 2017. 